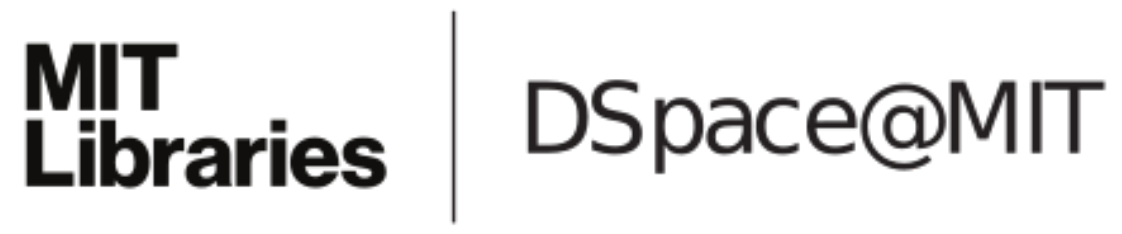

\author{
MIT Open Access Articles
}

\section{Observation of thermal occupation of room- temperature J-aggregate microcavity exciton-polaritons}

The MIT Faculty has made this article openly available. Please share how this access benefits you. Your story matters.

Citation: M. Scott Bradley and Vladimir Bulovic. "Conference on Lasers and Electro-Optics (CLEO) and Quantum Electronics and Laser Science Conference (QELS), 2010." IEEE. (C) Copyright 2010 IEEE

As Published: http://ieeexplore.ieee.org/xpls/abs_all.jsp?arnumber=5500669\&tag=1

Publisher: Institute of Electrical and Electronics Engineers (IEEE)

Persistent URL: http://hdl.handle.net/1721.1/74096

Version: Final published version: final published article, as it appeared in a journal, conference proceedings, or other formally published context

Terms of Use: Article is made available in accordance with the publisher's policy and may be subject to US copyright law. Please refer to the publisher's site for terms of use. 


\title{
Observation of Thermal Occupation of Room-Temperature J-Aggregate Microcavity Exciton-Polaritons
}

\author{
M. Scott Bradley*, Vladimir Bulović \\ Organic and Nanostructured Electronics (ONE) Lab \\ Massachusetts Institute of Technology, 77 Massachusetts Avenue, Cambridge, MA 02139 \\ email:sbradley@alum.mit.edu,bulovic@mit.edu
}

\begin{abstract}
We present a measurement of the lower-branch exciton-polariton occupation in roomtemperature J-aggregate microcavity devices under low-density steady-state excitation. The observed occupation follows a Maxwell-Boltzmann distribution at $\mathrm{T}=300 \mathrm{~K}$, indicating efficient polariton relaxation, necessary for achieving lasing.

(C)2010 Optical Society of America

OCIS codes: (240.5420) Polaritons; (160.4890) Organic materials
\end{abstract}

The use of organic materials in microcavity devices allows for the observation of the strong coupling of light and matter at room temperature due to the large oscillator strength and high binding energy of the Frenkel exciton transitions in organic molecular thin films [1,2]. In the past decade, research in the field of inorganic excitonpolariton devices has witnessed the demonstration of coherent light emission from exciton-polariton states, which may be a first indication of the superfluidity which has been theoretically predicted for microcavity excitonpolaritons; such polariton lasing, however, has remained elusive in organic systems, and experimental studies beyond linear optical measurements of organic microcavity devices have been minimal [3-8].

A key process in realizing exciton-polariton lasing is the relaxation of lower-branch exciton-polaritons from the exciton reservoir to the lowest energy in the lower-branch at $\mathrm{k}_{\|}=0\left(\theta=0^{\circ}\right)$ via scattering. In this study, we show a measurement of lower-branch exciton-polariton occupation in organic systems probed through photoluminescence (PL) and introduce a means of pumping the exciton-polariton states via a luminescent cavity spacer layer. The occupation of exciton-polaritons in the lower-branch in the measured devices is found to fit a Maxwell-Boltzmann (MB) distribution at $\mathrm{T}=300 \mathrm{~K}$, indicating that the population is thermalized. At higher pump intensities, we observe amplified spontaneous emission from the cavity spacer layer, indicating the potential of achieving lasing in an exciton-polariton mode via stimulated emission in the spacer layer.
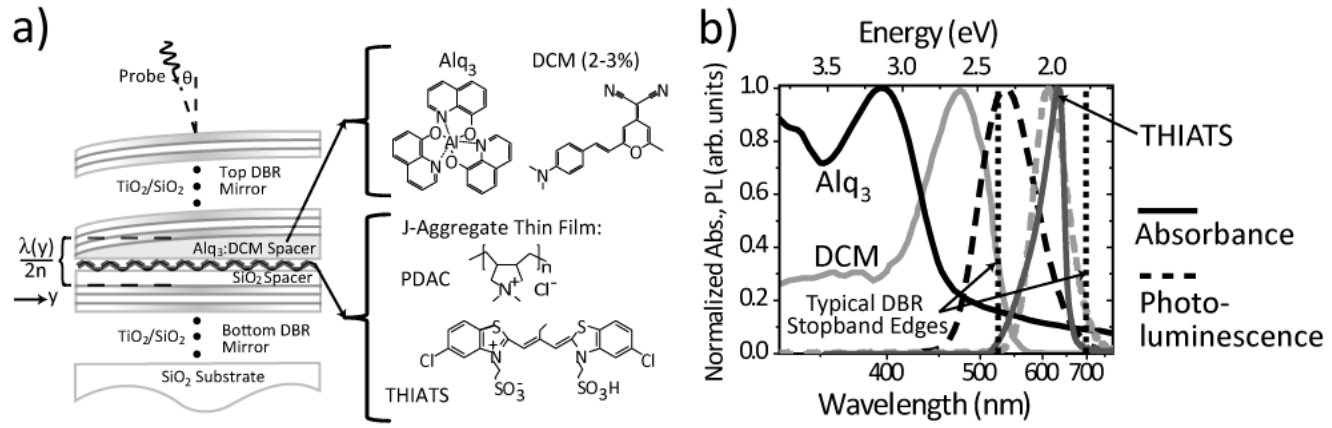

Figure 1: a) Device structure and molecular diagrams of constituent materials. b) Normalized absorbance and photoluminescence (PL) of constituent materials.

The structure of devices measured in this study is shown in Figure 1a. A microcavity is formed using two distributed Bragg reflectors (DBRs) consisting of alternating pairs of layers of silicon dioxide $\left(\mathrm{SiO}_{2}\right)$ and titanium dioxide $\left(\mathrm{TiO}_{2}\right)$. The J-aggregate thin film is deposited through spin self-assembly of alternating layers of PDAC [poly(diallyldimethylammonium chloride), MW 4-5 x 105, obtained from Sigma Aldrich, CAS 26062-79-3] and THIATS [Benzothiazolium, 5-chloro-2-[2-[[5-chloro-3-(3-sulfopropyl)-2(3H)-benzothiazolylidene]methyl]-1-buten1-yl]-3-(3-sulfopropyl)-, inner salt, compounded with N,N-diethylethanamine (1:1) obtained from H.W. Sands, CAS 23568-98-1]. After the J-aggregate thin film is deposited, a luminescent top cavity spacer of varying thickness is thermally evaporated consisting of $\mathrm{Alq}_{3}$ [aluminum tris(8-hydroxyquinoline)] doped 2-3\% with the laser dye DCM [4-(dicyanomethylene)-2-methyl-6-(4-dimethylaminostyryl)-4H-pyran]; the varying thickness is achieved by

\footnotetext{
* Present address: MIT Lincoln Laboratory, Lexington, MA.
} 


\section{QThM4.pdf}

evaporating the $\mathrm{Alq}_{3}$ :DCM thin film through a fixed shadow mask onto a rotating substrate. The use of a luminescent top cavity spacer allows for intracavity pumping of the THIATS J-aggregate microcavity excitonpolaritons, as shown by the absorption and PL spectra of the various materials in the microcavity in Figure 1b.

Figure 2 shows the angle-resolved PL collected from the sample shown in Figure 1a.
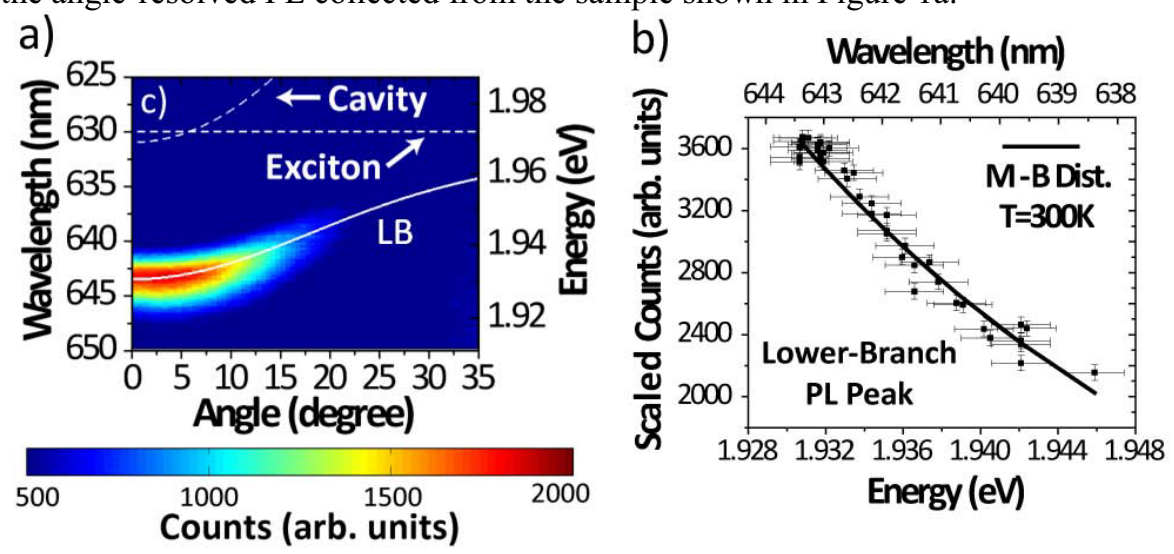

Figure 2: a) PL from lower-branch exciton-polariton vs. angle. b) Scaled PL counts from lower-branch exciton-polariton (relative occupation) vs. energy with Maxwell-Boltzmann fit at $\mathrm{T}=300 \mathrm{~K}$.

Figure 2a shows the angle-resolved PL at nearly-zero detuning. The measured lower-branch exciton-polariton PL is converted to a relative occupation number by taking account both of the effect of the planar structure on observed emission intensity and the effect of the changing composition of the exciton-polariton from photon to exciton due to larger positive detuning of the cavity resonance from the exciton resonance as the emission angle increases.

Figure $2 \mathrm{~b}$ shows the observed counts of the lower-branch exciton-polariton PL scaled to correct for the above effects with a plot of the $\mathrm{MB}$ distribution at $\mathrm{T}=300 \mathrm{~K}$. The excellent fit of the lower-branch exciton-polariton relative occupation to the $\mathrm{MB}$ distribution at $\mathrm{T}=300 \mathrm{~K}$ indicates that the exciton-polaritons pumped into the lowerbranch have thermalized prior to decay. Since the peak of the lower-branch exciton-polariton emission at $\lambda \sim 643 \mathrm{~nm}$ is considerably red-shifted from the DCM emission peak of $\lambda \sim 610 \mathrm{~nm}$, we conclude that the thermalization is likely occurring in the lower-branch exciton-polaritons and not in the DCM molecules prior to pumping the excitonpolaritons.

We have also observed that with increasing pump energy density, a threshold is seen in emission both from the lower-branch exciton-polariton and from the first Bragg mode of the DBR below the stopband, indicating the likely onset of amplified spontaneous emission in the luminescent cavity spacer layer. This result suggests that a type of "polariton laser" could be fabricated by making a VCSEL whose gain material is the luminescent cavity spacer layer and which lases in a strongly-coupled exciton-polariton mode. We will discuss threshold considerations for organic exciton-polariton lasers and the applicability of existing theory in inorganic exciton-polariton systems to organics.

[1] D. G. Lidzey, D. D. C. Bradley, M. S. Skolnick, T. Virgili, S. Walker, and D. M. Whittaker, "Strong exciton-photon coupling in an organic semiconductor microcavity," Nature, vol. 395, no. 6697, pp. 53-55, 1998.

[2] D. G. Lidzey, D. D. C. Bradley, T. Virgili, A. Armitage, M. S. Skolnick, and S. Walker, "Room temperature polariton emission from strongly coupled organic semiconductor microcavities," Phys. Rev. Lett., vol. 82, no. 16, pp. 3316-3319, 1999.

[3] J. R. Tischler, M. S. Bradley, Q. Zhang, T. Atay, A. Nurmikko, and V. Bulovic, "Solid state cavity qed: Strong coupling in organic thin films," Org. Electron., vol. 8, no. 2-3, pp. 94-113, 2007.

[4] R. J. Holmes and S. R. Forrest, "Strong exciton-photon coupling in organic materials," Org. Electron., vol. 8, no. 2-3, pp. 77-93, 2007.

[5] S. Kena-Cohen, M. Davanco, and S. R. Forrest, "Strong exciton-photon coupling in an organic single crystal microcavity," Phys. Rev. Lett., vol. 101, no. 11, p. 116401, 2008.

[6] J. Kasprzak, M. Richard, S. Kundermann, A. Baas, P. Jeambrun, J. M. J. Keeling, F. M. Marchetti, M. H. Szymanska, R. Andre, J. L. Staehli, V. Savona, P. B. Littlewood, B. Deveaud, and L. S. Dang, "Bose-einstein condensation of exciton polaritons," Nature, vol. 443, no. 7110, pp. 409-414, 2006.

[7] S. Utsunomiya, L. Tian, G. Roumpos, C. W. Lai, N. Kumada, T. Fujisawa, M. Kuwata-Gonokami, A. Loffler, S. Hofling, A. Forchel, and Y. Yamamoto, "Observation of bogoliubov excitations in exciton-polariton condensates," Nat. Phys., vol. 4, no. 9, pp. 700-705, 2008.

[8] A. Amo, D. Sanvitto, F. P. Laussy, D. Ballarini, E. del Valle, M. D. Martin, A. Lemaitre, J. Bloch, D. N. Krizhanovskii, M. S. Skolnick, C. Tejedor, and L. Vina, "Collective fluid dynamics of a polariton condensate in a semiconductor microcavity," Nature, vol. 457, no. 7227, pp. 291-U293, 2009. 